Rev. Elev. Méd. vét. Pays trop., 1974, 27 (1) : $39-52$

\title{
Recherche de l'activité immunostimulante de trois dérivés à action anthelminthique de l'imidazole sur les immunogenèses bovipestique, péripneumonique et charbonneuse
}

\author{
par A. PROVOST, G. TACHER et C. BORREDON (*) \\ (aide technique de Z. N'GALDAM, N. GOMBOT et D. ALLOUM)
}

\begin{abstract}
RESUME
Le but de l'expérience était de rechercher l'activité immunostimulante des anthelminthiques à noyau imidazole sur les immunogénèses bovipestique, péripneumonique et charbonneuse ( $B$. anthracis). Dans les conditions de la pratique (vaccination et droguage simultanés), aucun des trois produits ne montre d'activité immunostimulante statistiquement significative.
\end{abstract}

Une publication faite par G. et M. RENOUX en 1971 (21) ouvrait de grands espoirs d'application pratique : des souris recevant par injection sous-cutanée $25 \mu \mathrm{g}$ d'hydrochlorure de -d1- 2, 3, 5, 6 - tétrahydro -G- phénylimidazo$(2,1$ - b) - thiazole ou imidothiazole, corps de la chimie de synthèse à activité anthelminthique bien connue (Tétramisole, N.D.), 48 heures après avoir été vaccinées avec le vaccin antibrucellique Brucella abortus B 19, voyaient se développer chez elles une immunité antibrucellique stérilisante de même qualité, lors de l'épreuve virulente, que celle conférée par le vaccin antibrucellique $\mathbf{H} 38$ de RENOUX. En revanche, les souris vaccinées avec la seule souche B 19 hébergeaient un nombre variable de Brucella virulentes de la souche d'épreuve (**).

Chez l'homme, une expérience préliminaire des chercheurs du laboratoire Janssen Pharma-

(*) I.E.M.V.T., Laboratoire de Recherches Vétérinaires de Farcha, B.P. 433, N'Djamena, Tchad.

(**) Il nous est particulièrement agréable de remercier notre confrère et ami, le Pr. J. MORTELMANS, de l'Institut de Médecine tropicale d'Anvers, qui a attiré notre attention sur cette publication. ceutica à Beerse (Belgique) confirmait cette activité du tétramisole et de son isomère lévogyre sur l'immunogénèse anti-grippale: les sujets vaccinés avec un vaccin anti-grippal commercial qui absorbaient, pendant les 15 jours après la vaccination, du Tétramisole ou du 1-Tétramisole, avaient des titres en anticorps inhibant l'hémagglutination grippale doublés ou sextuplés par rapport aux sujets témoins n'ayant pas absorbé d'anthelminthique.

Enfin, des observations colligées en pratique vétérinaire indiquaient une activité immunostimulante du Tétramisole : chez des chiens traités pour ascaridiose et vaccinés contre la maladie de Carré, chez les bovins traités pour la bronchite vermineuse à Dictyocaulus et vaccinés contre la brucellose. Le traitement au Tétramisole dans cette dernière espèce semble même augmenter la résistance à la réinfestation naturelle par Dictyocaulus.

Ces résultats préliminaires étaient d'une application prometteuse en médecine vétérinaire tropicale. On pouvait espérer en effet que l'institution d'un droguage anthelminthique des bovins, appliqué par les équipes volantes de 
vaccination chargées de l'immunisation annuelle antibovipestique-antipéripneumonique, aurait un effet adjuvant sur l'immunogénèse, tout spécialement chez les veaux. On ferait ainsi d'une pierre deux coups.

Une expérience contrôlée a donc été montée pour vérifier, dans les conditions de la pratique de brousse, l'éventuel effet immunostimulant des anthelminthiques à noyau imidazole.

\section{MATERIEL ET METHODES}

\section{Généralités sur l'expérience}

On a voulu délibérément se placer dans les conditions d'une équipe de vaccination qui visite annuellement villages et campements de transhumance du Sahel, sans s'installer plus d'un jour ou deux à demeure; en d'autres termes, cela veut dire qu'elle ne dispose que d'une seule fois des animaux à vacciner, et éventuellement à traiter, et qu'en aucun cas elle ne peut pratiquer vaccination et droguage différés. On verra que cette condition, rendue impérative par l'ampleur des opérations annuelles de vaccination, est peut-être l'un des critères essentiels d'appréciation de l'expérience.

Le protocole, dès lors simple, consiste à vacciner des bovins dont on connaît le statut immunitaire vis-à-vis des antigènes qu'on leur inocule, à leur donner en même temps aux doses thérapeutiques les anthelminthiques choisis, puis à suivre la cinétique sérologique.

\section{Anthelminthiques essayés}

Etant donné les renseignements bibliographiques et les résultats déjà enregistrés, il tombe sous le sens que devaient être essayés le Tétramisole (corps racémique) et son isomère lévogyre, le Lévamisole (*). Un autre anthelminthique, le Thiabendazole, a une structure chimique voisine; il méritait lui aussi d'être essayé. Enfin, on pouvait se demander si l'effet immunostimulant enregistré avec le Tétramisole et le Lévamisole n'était pas corollaire de leur activité anthelminthique; pour vérifier le fait, un anthelminthique à large spectre d'activité mais à structure chimique différente, le tartrate de Pyrantel, est lui aussi mis en expérience.

(*) Notre confrère D. THIENPONT, de Janssen Pharmaceutica, a très aimablement mis à notre disposition $50 \mathrm{~g}$ de Lévamisole. Nous l'en remercions bien vivement.
On envisage donc d'avoir 4 groupes d'animaux vaccinés et drogués, et un groupe témoin qui reçoit le seul vaccin.

Les anthelminthiques ont été donnés aux doses thérapeutiques en médecine vétérinaire tropicale :

- Tétramisole sur la base de $10 \mathrm{mg} / \mathrm{kg}$ de poids vif chez les veaux et de $5 \mathrm{mg} / \mathrm{kg}$ pour les animaux plus vieux. Pour adapter la posologie à l'âge et au poids, on a utilisé des combinaisons de comprimés à 125 et $600 \mathrm{mg}$ vendus dans le commerce (Vadéphène, N.D. Spécia). Les comprimés étaient introduits dans le détroit pharyngé avec une longue pince à ceillères, suivi d'administration d'un peu d'eau à la bouteille.

- Lévamisole sur la base de $5 \mathrm{mg} / \mathrm{kg}$ de poids vif quel que soit l'âge. La préparation dont on disposait était sous la forme pharmaceutique de médecine humaine, incommode pour l'expérience, de comprimés à $50 \mathrm{mg}$. Les comprimés ont été broyés en poudre fine et celle-ci remise extemporanément en solution dans l'eau à raison de $5 \mathrm{~g}$ par litre. La posologie s'établissait donc à $100 \mathrm{ml}$ par $100 \mathrm{~kg}$ de poids vif, le médicament étant administré à la bouteille.

- Thiabendazole sur la base de 7,5 g par $100 \mathrm{~kg}$ de poids vif. On a utilisé la forme commerciale (Thibenzole, N.D. Merck, Sharp and Dohme) à 50 p. 100 de produit actif mélangé à une poudre mouillable. La suspension est préparée extemporanément et administrée à la bouteille.

- Tartrate de Pyrantel sur la base de $25 \mathrm{mg}$ par $\mathrm{kg}$ de poids vif. Parmi les préparations commerciales, on disposait de l'Exhelm (N.D. Pfizer) en solution toute prête, utilisable à la dose de $50 \mathrm{ml}$ par $100 \mathrm{~kg}$, donnée à la bouteille.

Les droguages ont été faits aussitôt après la vaccination individuelle des animaux.

\section{Vaccin}

L'intérêt de l'expérience étant de vérifier l'éventuel effet immunostimulant des anthelminthiques sur les antigènes utilisés dans la pratique, on fait appel à un vaccin de base pour la prophylaxie de masse : vaccin mixte trivalent antibovipestique - antipéripneumonie - anticharbon bactéridien récemment proposé $(17)$; 
il adjoint l'antigène bactéridien au vaccin mixte bien connu antibovipestique-antipéripneumonique $\left(^{*}\right)(18)$.

L'étude complète du vaccin trivalent (conception, production, contrôles) a été amplement exposée par ailleurs. On se rappellera que, conclusion décevante, ce vaccin mixte ne peut être d'application pratique au Tchad où la solution molaire de sulfate de magnésium est utilisée comme diluant des vaccins pestique et péripneumonique, faute de disposer de diluants réfrigérés sur le terrain; or, si la solution sulfatomagnésienne est thermoprotectrice pour les deux autres composants (15), elle se révèle délétère pour les spores charbonneuses, amenant une importante baisse de titre (17). Ce vaccin est donc réservé aux services vétérinaires utilisant la glace hydrique pour transporter les vaccins sur le terrain et refroidir les liquides de dilution.

Il a paru toutefois intéressant d'utiliser ce vaccin dans la présente expérience parce qu'il permettait l'étude de l'immunogénèse de trois antigènes. Des flacons d'un lot expérimental ont donc été transportés sur le lieu d'expérience en boîtes isothermes contenant de la glace fondante. La reconstitution et la dilution du vaccin ont été effectuées extemporanément, juste avant les opérations, en sérum physiologique glacé. Le vaccin reconstitué est conservé pendant la séance de vaccination en coffret isotherme contenant de la glace fondante. On injecte $1 \mathrm{ml} \mathrm{du}$ vaccin reconstitué par voie sous-cutanée rétroscapulaire.

Rappelons que les contrôles assignent les titres respectifs de $10^{3,2} \mathrm{DCP}_{50}$ pour le virusvaccin bovipestique, $9.10^{8}$ pour la péripneumonie, $7.10^{6}$ pour le charbon bactéridien.

\section{Animaux d'expérience}

En principe, l'immunisation annuelle légalement obligatoire au Tchad contre la peste bovine, et partant contre la péripneumonie par suite de la généralisation de l'utilisation du vaccin mixte antipestique-antipéripneumonique, rend alléatoire l'existence de bovins de plus d'un an réceptifs à ces deux maladies. En pratique, pour des raisons diverses, de tels animaux peuvent néanmoins être trouvés.

(*) Respectivement dénommés Trisec et Neobisec, produits par le Laboratoire de Farcha.
Le choix s'est porté sur deux campements reculés de l'ouest du Kanem, près de la frontière du Niger (puits de Foyo et Lalampui, à l'ouest de Nokou), endroit choisi parce que depuis 2 ans les tournées de vaccination n'avaient pu avoir lieu dans cette région. On pouvait donc s'attendre à trouver un certain nombre de bovins sans traces auriculaires de vaccination, donc vraisemblablement non vaccinés. Par ailleurs, la péripneumonie étant inconnue dans la région, on pouvait compter sur lïmmunogénèse de l'antigène péripneumonique pour se faire une opinion.

Deux cent trente quatre bovins âgés de 6 à 24 mois entrent en expérience (âge moyen en mois : $14,86 \pm 0,26$; intervalle de confiance à 95 p. 100). Avant la vaccination, ils subissent une prise de sang à la veine jugulaire avec l'appareil Phébo-pung (16) et reçoivent une boucle auriculaire numérotée. Aussitôt après la vaccination, ils sont drogués avec les anthelminthiques. On a ainsi 5 groupes d'animaux:

$$
\begin{aligned}
& \text { - groupe } 1 \text { (Lévamisole) : } 50 \text { animaux, } \\
& \text { - groupe } 2 \text { (Tétramisole) : } 50 \text { animaux, } \\
& \text { - groupe } 3 \text { (Thiabendazole) : } 40 \text { animaux, } \\
& \text { - groupe } 4 \text { (Pyrantel) : } 44 \text { animaux, } \\
& \text { - groupe } 5 \text {, témoin : } 50 \text { animaux. }
\end{aligned}
$$

Un prélèvement de matières fécales est effectué sur chaque veau et immédiatement mis dans des flacons répertoriés au numéro de l'animal, contenant du sérum physiologique formolé à 10 p. 100 . Les examens coprologiques sont faits au laboratoire.

Une seconde visite, donnant lieu à une prise de sang, a lieu 30 jours plus tard. On avait prévu d'apporter de la spiramycine (Suanovil, N.D. Spécia) pour éventuellement intervenir sur les réactions willemsiennes postvaccinales consécutives à l'inoculation de la souche $\mathrm{T}_{1}$-SR dc Mycoplasma mycoides dans une population bovine à réceptivité inconnue (7). Un certain nombre d'animaux n'ont pas été présentés lors de cette seconde visite car leurs propriétaires s'étaient déplacés. Par ailleurs, quelques tubes de prélèvements de sang se sont renversés au cours du transport, si bien que l'on n'a pas disposé au laboratoire de la totalité des 234 paires de sérum, comme l'indiquent les tableaux I à $\mathrm{V}$. Il en a été tenu compte dans l'interprétation statistique des résultats. 
TABLEAU $\mathrm{N}^{\bullet} \mathrm{I}$

Groupe 1 - Leyamisole (dose $5 \mathrm{mg} / \mathrm{kg}$ - solution $5 \mathrm{~g} / 1$ )

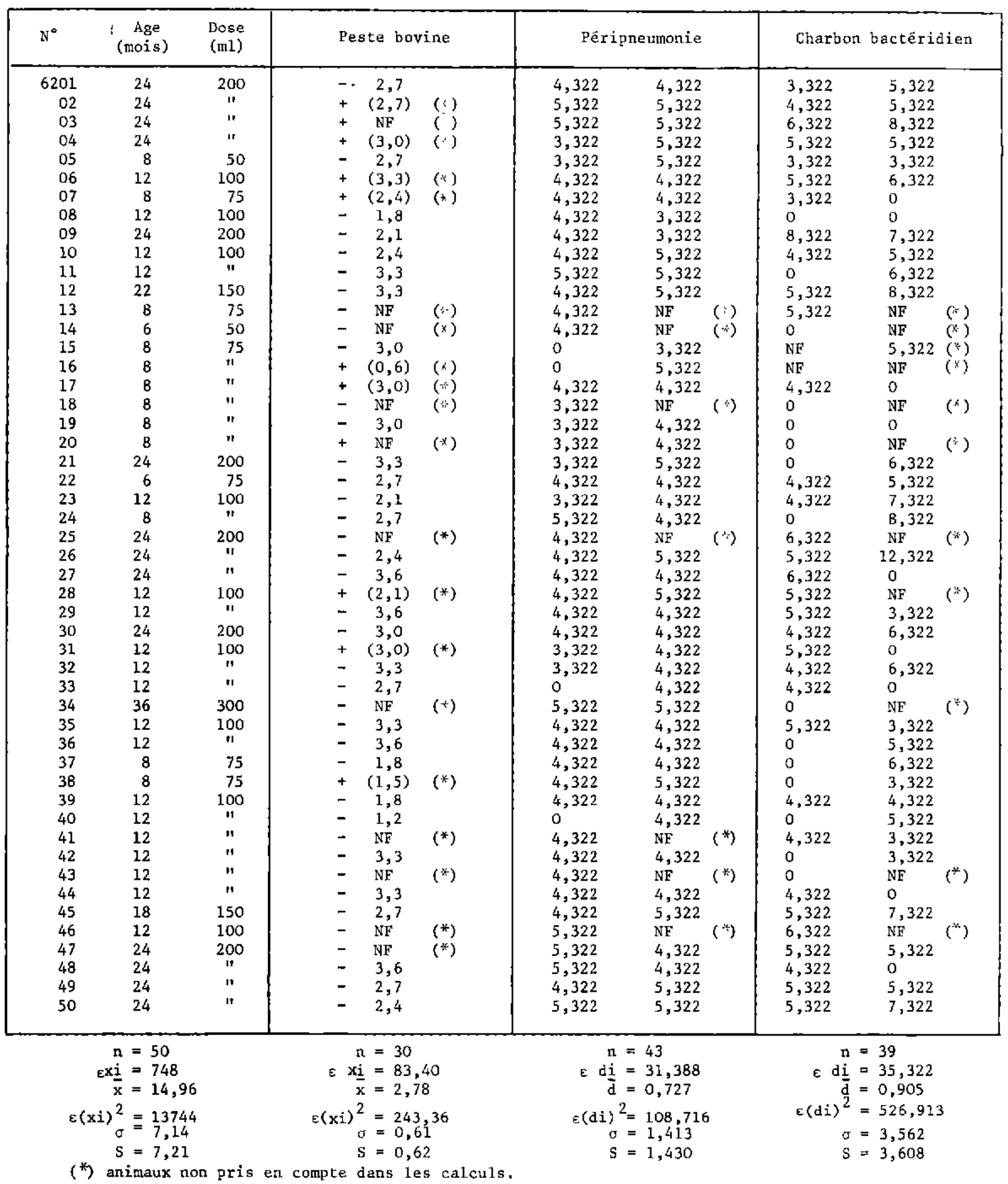


TABLEAU $N^{\circ}$ II

Groupe 2 - Tétramigole (dose : $10 \mathrm{mg} / \mathrm{kg}$ )-Comprimés à $600 \mathrm{mg}$ )

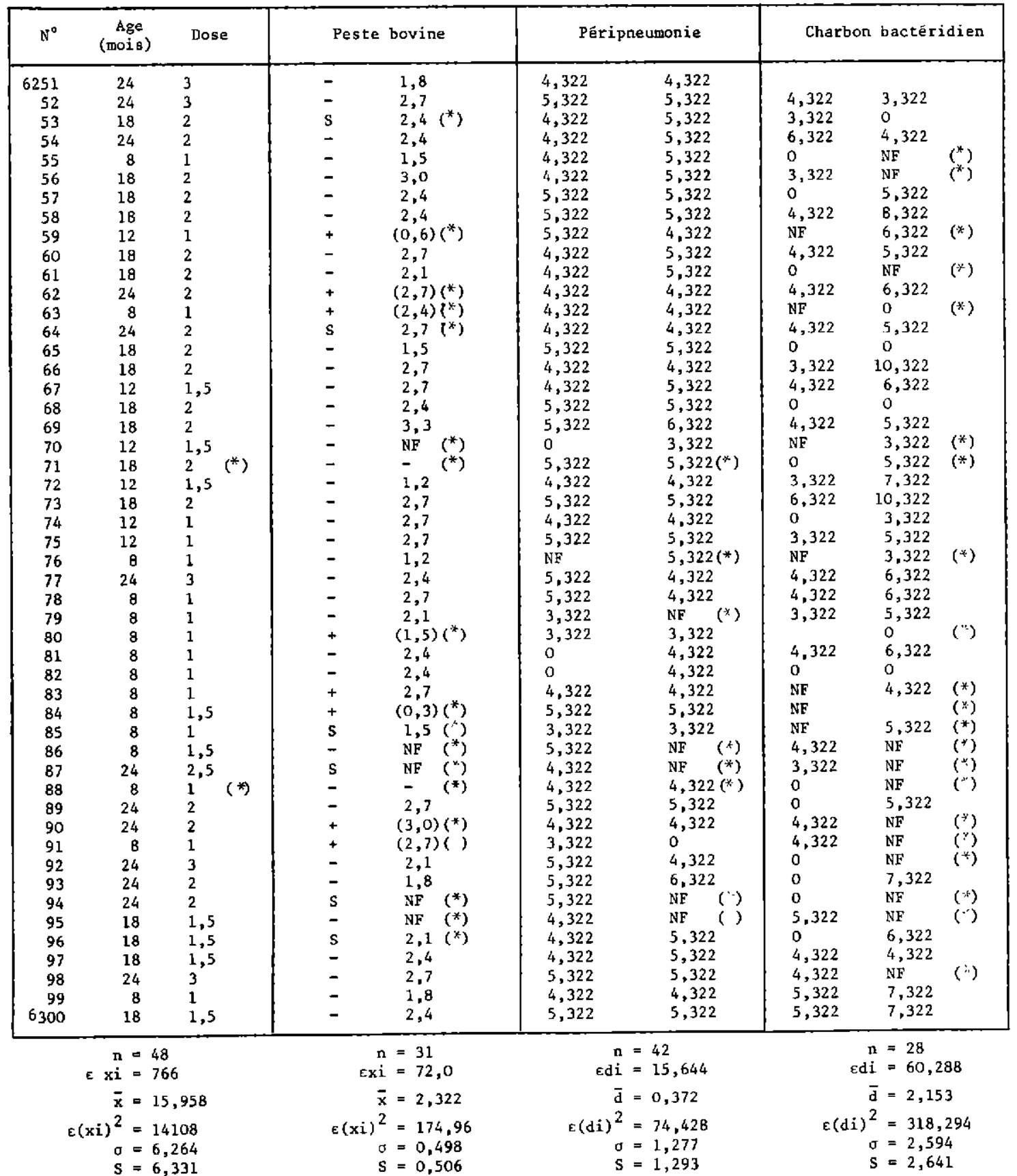

(*) animaux non pris en compte dans les calculs. 


\section{Techniques sérologiques}

\section{a) Anticorps antibovipestiques}

Ils sont mesurés par séro-neutralisation quàntitative (virus constant - sérum variable), en" cultures cellulaires de rein d'embryon de veau selon la technique de PLOWRIGHT et FERRIS (11). Leur titre est exprimé par l'indice $\mathrm{TN}_{50}$ (titre neutralisant 50 p. 100) sur la base de l'exposant du $\log _{10}$ de la dilution de sérum.

\section{b) Anticorps péripneumoniques}

On fait appel à une technique de fixation du complément (technique de Farcha) dérivée de la technique de KOLMER (20). Quoique entachée de non-spécificité dans les basses dilutions des sérums examinés, elle a le mérite d'être particulièrement sensible, donc de se prêter à la mesure de la faible conversion sérologique induite par la souche $\mathrm{T}_{1}-\mathrm{SR}$ de Mycoplasma mycoides (6). Les titres sont exprimés par l'exposant du $\log _{2}$ de la dilution de sérum donnant une fixation d'au moins 75 p. 100.

\section{c) Anticorps anticharbonneux}

La recherche de ces anticorps n'est pas une opération classique en laboratoire. Dans le cadre de la présente expérience, où l'on voulait

TABLEAU $N^{\circ}$ III

Groupe 3 - Thiabendazole (dase $7.5 \mathrm{~g} / 100 \mathrm{~kg}$, soit $50 \mathrm{ml} / 100 \mathrm{~kg}$ )

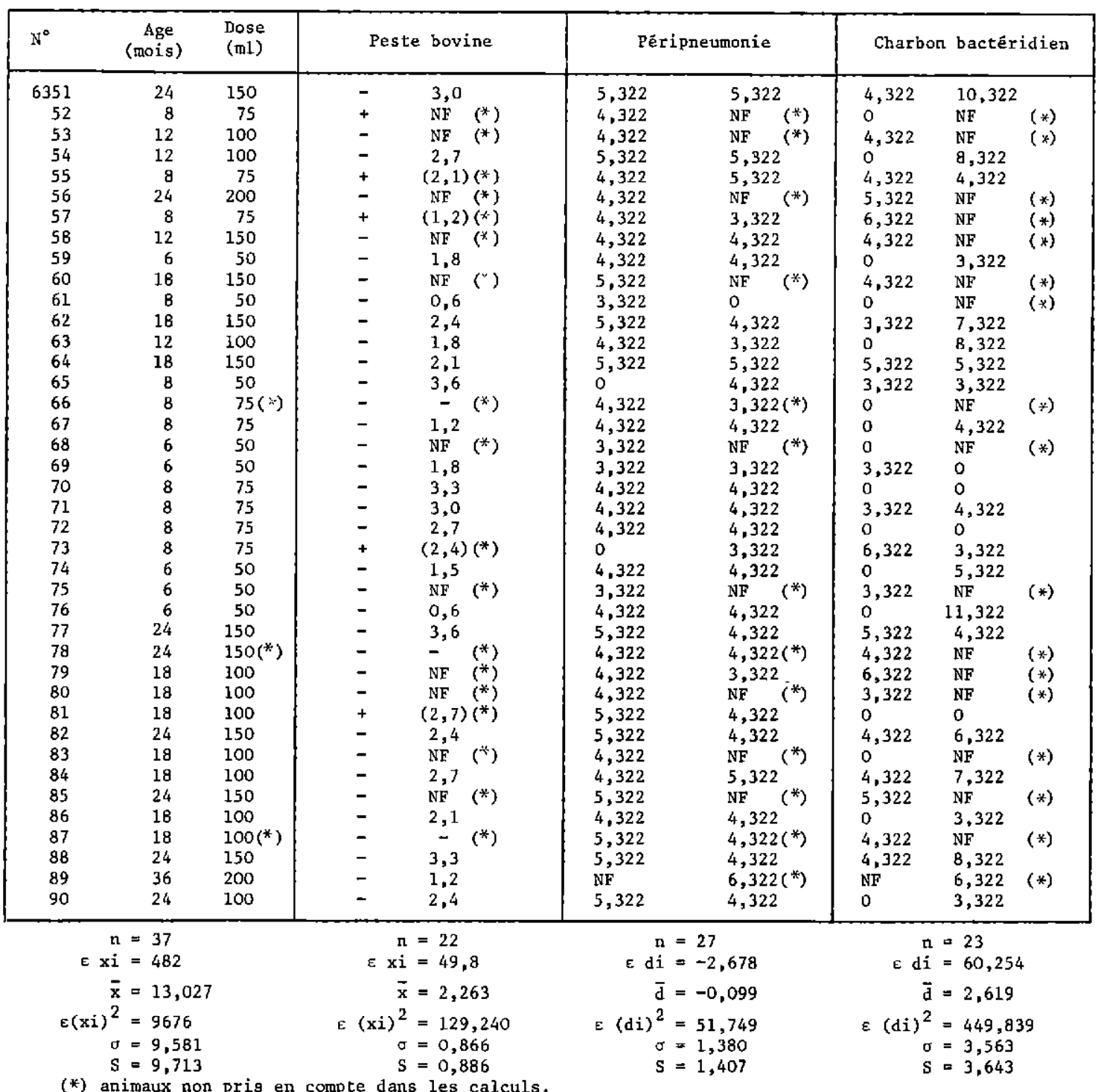


mesurer une immunogénèse, elle a pourtant été envisagée car elle permettait l'étude d'un paramètre supplémentaire.

La fixation du complément n'a pas, pour cette recherche, la faveur des expérimentateurs par suite d'erreurs par défaut (1). La technique récente proposée par $\mathrm{CHO}(5)$ peut souffrir du même reproche puisqu'elle détecte la même classe d'anticorps. Pour ces raisons, on a pensé utiliser la technique d'hémagglutination indirecte qui paraît détecter, selon BUCHANAN et collab. (1), les anticorps protecteurs chez les vaccinés.

Un premier antigène est préparé par sensibilisation d'hématies de mouton, formolées selon la technique de CARTER et RAPPAY (4), avec une suspension en sérum physiologique formolé à 1,5 p. 100 d'une culture de 24 heures sur gélose de Bacteridium anthracis souche Sterne 34F2, donc récoltée avant que la sporulation ne soit effective. Essayé avec différents sérums de moutons ayant servi aux

TABLEAU $\mathrm{N}^{\circ}$ IV

Groupe 4 - Tartrate de Pyrantel (dose $50 \mathrm{ml} / 100 \mathrm{~kg}$ )

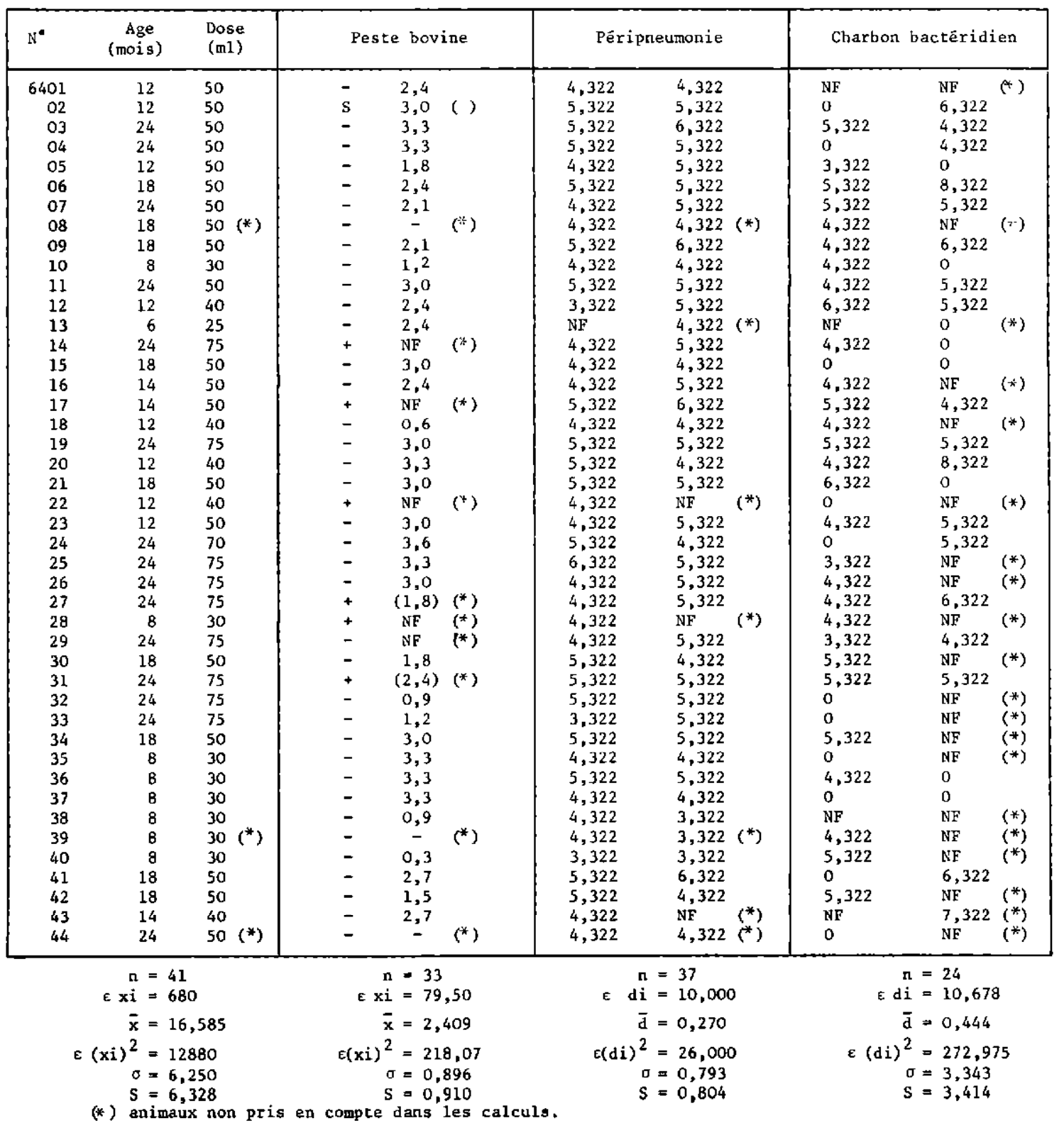


TABLEAU $\mathrm{x}^{\circ} \mathrm{V}$

Groupe 5 - Témoin.

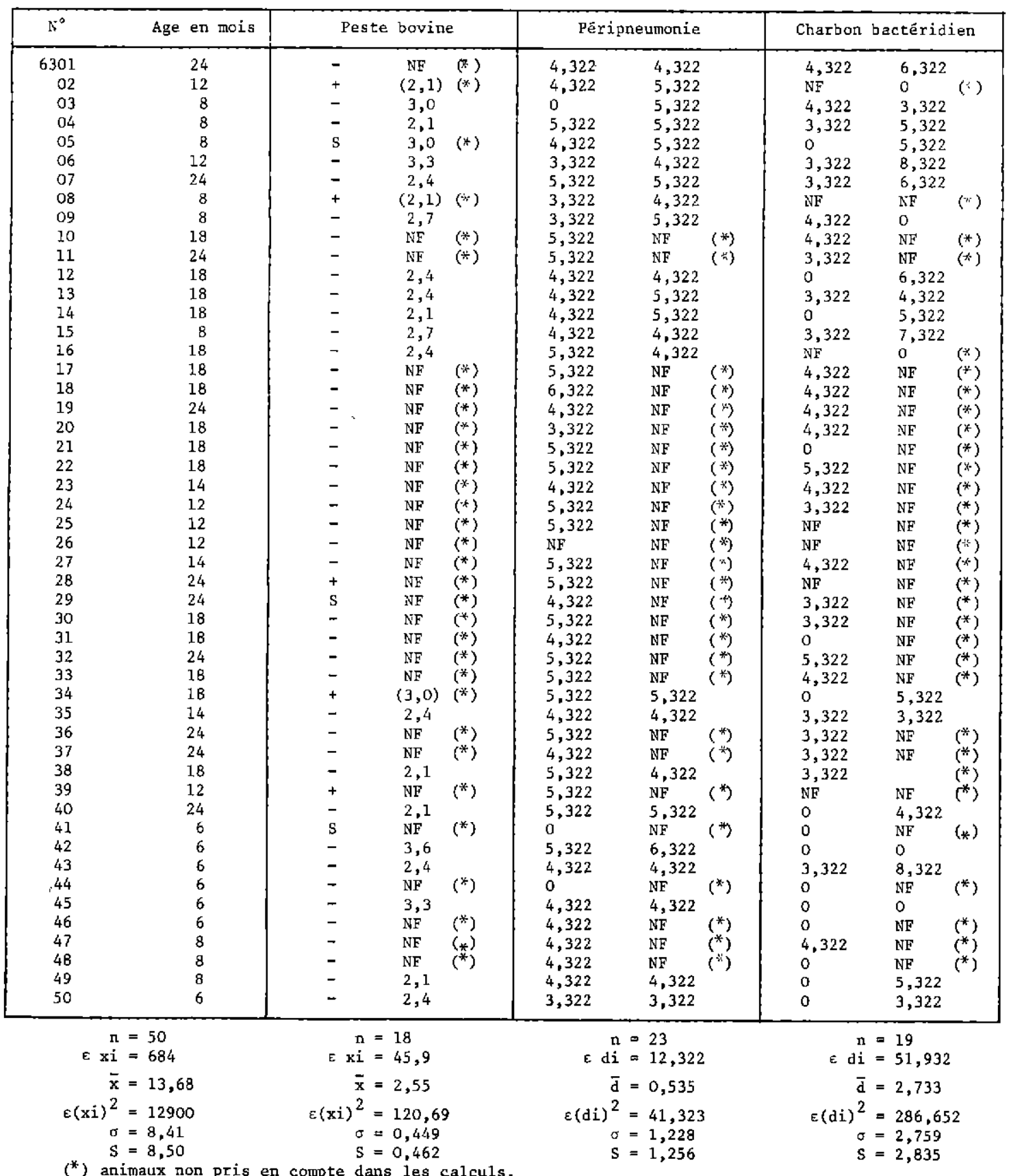


tests d'innocuité du vaccin anticharbonneux préparé au laboratoire, il ne s'est pas révélé satisfaisant par manque de sensibilité.

On a alors préparé un antigène inspiré de celui de PALIT et NILAKANTAN (9), consistant en une suspension d'hématies sensibilisées par un complexe polyosidique extrait d'une culture de la bactéridie. A cet effet, on récolte en sérum physiologique une culture de 12 heures sur gélose à 3 p. 100 d'extrait de levure (donc non sporulée) de la souche $34 \mathrm{~F} 2$. Le complexe polyosidique somatique est extrait selon la technique de WESTPHAL du phénol à chaud, en mettant en cuvre la méthodologie que BUTTERY et PLACKETT (2) ont utilisée pour la galactane péripneumonique. Utilisé à la concentration de $0,1 \mathrm{mg} / \mathrm{ml}$, il sert à sensibiliser une suspension à 0,25 p. 100 d'hématies de chèvre formolées. La chèvre a été choisie comme espèce donatrice d'hématies car rares sont les sérums de zébu possédant des hétéroagglutinines naturelles dirigées contre elles (19). Après plusieurs lavages pour éliminer l'excès de polyoside, les hématies sensibilisées sont remises en suspension à 0,25 p. 100 dans un tampon phosphaté à $\mathrm{pH}: 7$ contenant 0,1 p. $100 \mathrm{de}$ gélatine et 0,5 p. 100 d'albumine bovine comme stabilisateurs.

Le test est très simplement exécuté par examen de dilutions en progression géométrique de raison 2 des sérums sous test: mélange de $0,4 \mathrm{ml}$ de chaque dilution avec $0,4 \mathrm{ml}$ de la suspension d'hématies sensibilisées et lecture après un séjour de 2 heures à la température du laboratoire. Des témoins sont inclus pour apprécier la stabilité de l'antigène et l'absence d'hétéro-agglutinines dans les sérums. Les titres sont exprimés par l'exposant du $\log _{2}$ de la dilution de sérum donnant une hémagglutination d'au moins 75 p. 100.

\section{RESULTATS}

\section{Comportement clinique des animaux vaccinés}

La vaccination avec le vaccin mixte trivalent est parfaitement inoffensive. On n'a rapporté aucune réaction générale ni noté de réaction locale, ce qui atteste l'innocuité de la souche $\mathrm{T}_{1}$-SR de $M$. mycoides pour les veaux de race zébu arabe et de l'association vaccinale en général. On avait en effet émis des craintes, toutes théoriques, au souvenir de l'observation de MORNET, ORUE et DIAGNE (8) sur l'apparition de lésions willemsiennes consécutives à l'inoculation sous-cutanée d'une souche atténuée de $M$. mycoides suivie de celle d'un vaccin anticharbonneux. C'est pour cette raison que l'on avait prévu d'intervenir éventuellement avec la spiramycine.

Du même coup est confirmée, s'il en était besoin, l'innocuité de la vaccination couplée au droguage anthelminthique. La double opération est donc parfaitement licite, d'autant que les composants de l'imidazole à activité anthelminthique ne possèdent aucune activité antimycoplasmique ou antibactérienne pouvant interférer avec l'immunogénèse $(13,26)$.

L'infestation vermineuse du groupe de veaux à cette époque de l'année (janvier) était extrêmement faible. On n'en a trouvé que 10 qui étaient porteurs de strongylidés (36 à 180 cufs par gramme de fèces) $\left({ }^{*}\right)$. L'effet anthelminthique des médicaments sera donc à éliminer de l'interprétation des résultats.

\section{Résultats sérologiques}

Les résultats sérologiques d'ensemble figurent dans les tableaux $\mathrm{I}$ à $\mathrm{V}$ où ils sont groupés par médicament essayé. Ainsi qu'on l'a dit, un certain nombre de sérums manquent pour la deuxième saignée. Le signe $(*)$ indique dans les tableaux les animaux ou les paires de sérum qui ont été exclus des calculs statistiques.

\section{a) Immunogénèse bovipestique}

Sur 234 veaux disponibles lors de la vaccination, on en trouve 35 qui possèdent des anticorps bovipestiques neutralisants : 19 sont âgés de 12 mois et plus, 16 de moins de 12 mois et, en général, de 8 . On remarque toutefois qu'aucun des jeunes veaux de 6 mois n'a d'anticorps, que l'on aurait pu penser, vu leur âge, être d'origine colostrale. On est alors conduit à se demander quelle peut être l'origine de tels anticorps neutralisants chez des animaux sans trace auriculaire de vaccination : contact fortuit avec des souches bovipestiques hypovirulentes ou vaccination méconnue des propriétaires par suite d'échanges coutumiers d'animaux et sans marque auriculaire apposée ? Il est impossible

(*) Nous remercions notre collègue P. M. TRONCY qui a pris le soin de ces examens. 
de répondre. Pour l'appréciation de l'immunogénèse bovipestique, ces 35 animaux ont été exclus du calcul.

D'autre part, les sérums souillés soit avant soit après vaccination ont également été exclus des calculs.

Sur 142 paires de sérums sans anticorps antipestiques antevaccinaux utilisables, on constate une conversion sérologique pour 134 d'entre eux. Huit animaux de tous âges $\left(\mathrm{n}^{\circ} 6271,6288\right.$, $6366,6378,6387,6408,6439$ et 6444) ne répondent pas à la vaccination. Comme la vaccination antipestique chez un bovin réceptif est toujours suivie d'une conversion sérologique et considérant que pour les autres antigènes ces animaux n'ont pas non plus montré de conversion sérologique positive, on est conduit à penser que l'inoculation vaccinale, pratiquée dans des conditions de brousse avec contention sommaire, a été mal faite. Ils seront donc exclus $\mathrm{du}$ calcul statistique. Le titre moyen $\mathrm{TN}_{50}$ des anticorps postvaccinaux dans l'ensemble des bovins est de 2,47 $\pm 0,12$ (intervalle de confiance de la moyenne donné avec une probabilité de 95 p. 100; c'est celui qui sera donné par la suite).

Si maintenant on calcule le $\mathrm{TN}_{50}$ par groupe, on obtient les chiffres suivants :

$$
\begin{aligned}
& \text { Groupe } 1: 2,78 \pm 0,23 \\
& \text { Groupe 2: } 2,32 \pm 0,19 \\
& \text { Groupe } 3: 2,26 \pm 0,39 \\
& \text { Groupe } 4: 2,41 \pm 0,32 \\
& \text { Groupe } 5: 2,55 \pm 0,23 .
\end{aligned}
$$

Un test d'analyse de variance montre qu'il n'y a aucune différence significative entre les différents groupes dans la montée d'anticorps après vaccination $(F=1,593)$.

\section{b) Immunogénèse antipéripneumonique}

On dispose de 172 paires de sérums. Cent soixante deux animaux présentent avant vaccination des anticorps fixant le complément (technique de Farcha) à faible titre; cette situation est normale avec le type de réaction choisie, de grande sensibilité, et ne signe nullement une contamination péripneumonique.

Les conversions sérologiques après vaccination sont fort inconstantes et de faible intensité : elles ne touchent que 59 animaux et n'interviennent que pour 1 ou 2 dilutions des sérums.
De plus, 26 sérums voient leur titre baisser de la première à la deuxième saignée.

En calculant les moyennes des montées d'anticorps par groupe, on obtient les chiffres suivants :

$$
\begin{array}{ll}
\text { Groupe 1: } & 0,727 \pm 0,44 \\
\text { Groupe 2: } & 0,372 \pm 0,40 \\
\text { Groupe 3: } & -0,099 \pm 0,56 \\
\text { Groupe } 4: & 0,270 \pm 0,27 \\
\text { Groupe } 5: & 0,535 \pm 0,54 .
\end{array}
$$

Un test d'analyse de variance montre qu'il n'y a aucune différence significative entre les différents groupes dans la montée du titre des anticorps après vaccination $(F=0,384)$.

Incidemment, il n'y a pas à être surpris de l'inconstance et de la faiblesse de la conversion sérologique post-vaccinale; c'est un fait déjà établi avec la souche $\mathrm{T}_{1}$ de $M$. mycoides et qui est encore plus net avec la souche $T_{1}-S R(6)$. On ne doit en tirer aucune conclusion défavorable quant à l'immunité subséquente.

\section{c) Immunogénèse charbonneuse}

On possède 133 paires de sérums. Avant vaccination, 89 d'entre eux (67 p. 100) possèdent des anticorps actifs en hémagglutination indirecte.

Il paraît difficile d'apporter une explication à leur présence : manque de spécificité de l'antigène mis en œuvre, contact antérieur avec l'antigène charbonneux ou avec des bactéries à antigénicité proche? Dans cet ordre d'idées, une vaccination anticharbonneuse paraît devoir être exclue. En effet, elle aurait été faite par une équipe volante de vaccination qui aurait vacciné en même temps contre la peste. Or les anticorps anticharbonneux ne se retrouvent pas, et de loin, chez tous les animaux à sérologie bovipestique antevaccinale positive et nombreux sont ceux à sérologie bovipestique négative qui hébergent des anticorps charbonneux. Par contre, on remarque qu'il y a une corrélation hautement significative entre les titres des anticorps charbonneux avant vaccination et l'âge des animaux. En appelant y le log. du titre et $x$ l'âge des animaux en mois, la droite de régression a pour équation :

$$
\mathrm{y}=0,10 \mathrm{x}+1,488
$$

le coefficient de régression étant très hautement significatif ( $t$ de Student pour 131 degrés de liberté $=3,44$ ). Il est donc possible qu'il y 
ait sensibilisation progressive des animaux au cours de leur vie par des antigènes des bacilles anthracoïdes, dont Bacillus cereus qui possède une polyoside somatique d'activité antigénique proche de celle de $B$. anthracis.

La vaccination est suivie d'une montée d'anticorps chez 81 animaux (61 p. 100); 25 sérums ont des titres identiques avant et après vaccination (12 négatifs, 13 avec des titres variables) et 27 accusent une baisse de titre. Les moyennes des montées d'anticorps par groupe s'établissent ainsi :

$$
\begin{aligned}
& \text { Groupe } 1: 0,905 \pm 1,17 \\
& \text { Groupe } 2: 2,153 \pm 1,23 \\
& \text { Groupe } 3: 2,619 \pm 1,58 \\
& \text { Groupe } 4: 0,444 \pm 1,44 \\
& \text { Groupe } 5: 2,733 \pm 1,37 .
\end{aligned}
$$

Un test d'analyse de variance montre une différence à la limite de la signification entre les groupes (pour $\delta_{1}=4, \delta_{2}=128$ on trouve $F=2,476$, alors que les tables donnent une signification à 95 p. 100 à partir de 2,45 ); seul le lot 4 est d'ailleurs significativement différent du lot 5 et 3 .

\section{DISCUSSION}

De l'ensemble des résultats exposés, on tire la première conclusion que le vaccin mixte antibovipestique - antipéripneumonique - anticharbonneux a joué son rôle d'immunigène. $\mathrm{Ce}$ point a déjà été discuté par ailleurs (17). La seconde conclusion, que l'on a déjà formulée, est la possibilité du droguage anthelminthique pratiqué en même temps que la vaccination. Comme on a déjà montré (14) que l'on pouvait impunément inoculer un trypano-préventif à noyau métamidium (Ethidium, N.D. Boots Pure Drugs; Trypamidium, N.D. Spécia) sans effet fâcheux pour l'immunogénèse, on arrive ainsi à d'importantes simplifications de la prophylaxie de masse.

L'interprétation de l'effet immunostimulant des médicaments anthelminthiques est basée sur une étude de la cinétique sérologique. $\mathrm{Ce}$ point mérite discussion.

Si l'on accepte, en toute logique, que les huit veaux qui n'ont montré aucune montée d'anticorps antipestiques sont des ratés lors de linjection du vaccin, on ne trouve aucune critique pour les titres en anticorps pestiques enregistrés; ils sont d'excellente qualité lorsqu'on les compare à d'autres résultats utilisant la même technique de séro-neutralisation $(12$, 23).

Pour l'étude des anticorps péripneumoniques, on a fait le choix de la réaction de fixation du complément, technique de Farcha, beaucoup plus sensible que la classique réaction de CAMPBELL et TURNER (3). La réaction d'hémagglutination conditionnée (10), elle aussi particulièrement sensible, a été écartée après essai par suite de l'inconstance trop flagrante de la détection des anticorps chez les vaccinés. Pourtant, même avec la réaction de Farcha, on est loin d'enregistrer des conversions sérologiques nettes après vaccination puisqu'on n'a que 34,3 p. 100 des vaccinés qui exhibent une montée d'anticorps. C'est au demeurant, on l'a déjà souligné, une situation normale avec la souche $\mathrm{T}_{1}-\mathrm{SR}$ de $M$. mycoides, qui en fait se révèle précieuse sur le plan pratique puisque la vaccination ne vient pas troubler les opérations de prophylaxie sanitaire basées sur l'identification sérologique des malades. Pour le point qui nous occupe, celui de la recherche d'un éventuel effet immunostimulant des anthelminthiques à noyau imidazole, les résultats sérologiques enregistrés conservent tout de même leur valeur en donnant un paramètre de plus à mesurer et à interpréter, sans que l'on soit pour autant autorisé à en tirer des conclusions quant à la solidité de l'immunité péripneumonique subséquente.

La mesure des anticorps anticharbonneux a fait appel à une réaction d'hémagglutination conditionnée, réputée fournir une indication des anticorps protecteurs (1). La seule publication dont nous ayons connaissance ayant utilisé cette réaction en médecine vétérinaire est celle de PALIT et NILAKANTAN (9). On constate qu'eux aussi détectent des anticorps hémagglutinants dans les sérums * normaux * avant vaccination. L'explication apportée plus haut paraît être convenable et l'on est en droit de se demander s'il n'y aurait pas lieu de faire appel à une autre fraction antigénique de $B$. anthracis ou de ses métabolites; dans cet ordre d'idée, la sensibilisation d'hématies par l'antigène * protecteur $\gg$ du microbe charbonneux (24) mériterait d'être étudiée. Qu'elle soit spécifique ou non, la réaction d'hémagglutination que nous avons employée se révèle décevante à l'analyse par manque de sensibilité, ce qui au demeurant 
n'influe pas sur la conclusion générale de l'expérience étant donné l'hypothèse d'une activité immunostimulante nette des anthelminthiques à noyau imidazole; si elle avait existé, les titres en anticorps auraient pallié ce défaut de sensibilité.

La conclusion générale de l'essai est en effet très réservée quant à l'éventualité d'une activité immunostimulante des médicaments essayés. On concèdera pourtant que le Lévamisole semble posséder une telle propriété, dans les conditions de l'essai, sur l'immunogénèse bovipestique, sans pourtant que le résultat soit significatif. Il est possible que pour l'antigène péripneumonique, les résultats aient été plus significatifs si la seconde prise de sang était intervenue 18 à 21 jours après la vaccination; on connaît en effet la fugacité des anticorps fixant le complément engendrés par la souche $\mathrm{T}_{1}$-SR. Pour des raisons matérielles, il n'était pas possible de faire autrement que ce qui a été décrit dans le protocole expérimental. Au demeurant, répétons-le, les anticorps fixant le complément ne sont pas, dans la péripneumonie, le reflet de la résistance à la maladie, résistance dont les facteurs restent toujours inconnus.

L'action immunostimulante qui a été recherchée concerne, pour la peste bovine et le charbon bactéridien, une immunité protectrice par anticorps sériques. Or, dans l'expérience princeps des RENOUX (21), c'est une immunité cellulaire par immunphagocytes qui a été testée. L'origine de ces deux immunités est différente, tout comme leur mécanisme. Si l'on évoque des travaux non publiés sur l'activité du Lévamisole et du Tétramisole chez des cancéreux, on penche plutôt pour l'opinion que ces deux anthelminthiques favorisent l'immunité cellulaire mais non l'immunité sérique par anticorps circulants (25). Pourtant, une récente expérience de G. et M. RENOUX (22) permet de conclure que leur activité immunostimulante joue chez la souris pour les cellules spléniques immunocompétentes, tout au moins dans la production des IgM, seules immuno-globulines étudiées. Par ailleurs, l'activité est bien plus nette lorsque les deux drogues sont inoculées 18 heures après l'injection de l'antigène que lorsqu'elles sont injectées en même temps que lui.

Il est possible que le moment d'application des anthelminthiques par rapport à celui de l'injection des antigènes soit un facteur critique; cela ressort nettement des deux expériences de RENOUX, tout comme de celle des chercheurs de Janssen Pharmaceutica évoquée dans l'introduction : l'effet immunostimulant est très net lorsque les médicaments sont donnés en un temps ultérieur à celui des antigènes. En ce qui nous concerne, nous avons voulu nous mettre dans les conditions pratiques du travail de brousse, où les équipes de vaccination ne disposent que d'une seule fois des animaux, donc doivent réaliser en un seul temps vaccination et droguage. Il est parfaitement illusoire de vouloir pratiquer autrement, car cela reviendrait à doubler les équipes, les moyens et le coût de l'intervention, nécessitant de surcroît une nouvelle contention des animaux, opération pénible dans le Sahel et qui n'est jamais sans danger ni pour les animaux ni pour les éleveurs. Dans la pratique, on ne peut donc songer qu'à l'application simultanée de la vaccination et du droguage.

La pratique de la vaccination doublée du droguage anthelminthique paraît être recommandable pour autant que l'intensité de parasitisme la nécessite. Ce faisant, on conservera l'espoir de voir certaines immunités, dont peutêtre l'immunité bovipestique et vraisemblablement l'immunité antibrucellique là où la vaccination est instituée, être renforcées par le Lévamisole.

\section{NOTE}

Cet article était déjà rédigé lorsque nous avons eu connaissance de la publication de V. B. KULKARNI et collab. (Ind. vet. J., 1973, 50, 225-227). Ces auteurs détectent chez les poulets vaccinés contre la maladie de Newcastle (souche La Sota) à l'âge de 2 jours puis drogués au Tétramisole à la dose de $1 \mathrm{mg} / 50 \mathrm{mg}$ de poids vif pendant 3,7 et 15 jours, une montée d'anticorps inhibant l'hémagglutination légèrement supérieure à celle des témoins ne recevant pas de Tétramisole. 


\section{SUMMARY}

\section{Research on the immunostimulating capability of three imidazole derivatives with anthelmintic activity on the rinderpest, CBPP and anthrax immunogenesis}

The aim of the experiment was to investigate the potentiating capabilities on rinderpest, bovine pleuropneumonia and anthrax immunogenesis of anthelminthic drugs with imidazole nucleus. In field conditions, they have been found to be significantly devoided of immunostimulating activity.

\section{RESUMEN}

\section{Busqueda de la actividad inmunoestimulante de tres derivados del Imidazole con acción antihelmíntica sobre los inmunogenesis bovipestica, perineumonica $\mathbf{y}$ carbuncosa}

La experiencia tenia por objeto de buscar la actividad inmunoestimulante de los antihelmínticos con núcleo imidazole sobre las inmunogenesis bovipestica, perineumonica y carbuncosa (B. anthracis).

En las condiciones de la práctica (vacunación y administración de medicamentos simultáneas) ningún de los tres productos muestra una actividad inmunoestimulante estadísticamente significativa.

\section{BIBLIOGRAPHIE}

1. BUCHANAN (T. M.), FEELEY (J. C.), HAYES (P.S.) et BRACHMAN (P.S.). Anthrax indirect microhemagglutination test. J. Imm., 1971, 107 : 1631-1636.

2. BUTTERY (S. H.) et PLACKETT (P.). A specific polysaccharide from Mycoplasma mycoides. J. gen. Microbiol., 1960, 23 : 357-368.

3. CAMPBELL (A.D.) et TURNER (W.). Studies on contagious pleuropneumonia of cattle. IV. An improved complement fixation test. Aust. vet. J., 1953, 29 : 154-163.

4. CARTER (G. R.) et RAPPAY (D. E.). Formalinized erythrocytes in the haemagglutination test for typing Pasteurella multocida. Brif. vet. J., 1962, 118: 289-292.

5. CHO (H. J.). Demonstration of complement fixing antibody in the sera of cattle vaccinated with combined living blackleg-anthrax vaccine Canad. J. comp. Med., 1971, 35: 155-160.

6. DOUTRE (M. P.), CHAMBRON (J.) et BOURDIN (P.). Valeur de l'immunité conférée par un vaccin mixte antibovipestique-antipéripneumonique lyophilisé préparé à l'aide de la souche $T_{1}-S R$. Rev. Elev. Méd. vét. Pays trop., 1972, 25 : 1-14.

7. LINDLEY (E. P.). La spiramycine et les lésions post-vaccinales au vaccin lyophilisé $M$. mycoides var. mycoides souche $\mathrm{T}_{1} / 44$ contre la péripneumo. nie contagieuse des bovidés. Cah. Méd. vét., 1971, 40: 233-236.

8. MORNET (P.), ORUE (J.) et DIAGNE (L.). Persistance in vivo, dans le tissu conjonctif souscutané, du virus pérıpneumonique et vaccinations différées avec des vaccins vivants. Bull. Acad. vét. Fr., 1947, $20: 467-471$.

9. PALIT (A.) et NILAKANTAN (P. R.). Antibody response in animals immunized with different types of anthrax vaccine. Ind. J. vet. Sci., 1968, 38 : 431-442.

10. PERREAU (P.), PROVOST (A.), REGNOULT (R.) et ORUE (J.). Valeur de la réaction d'hémagglutination indirecte dans la péripneumonie bo- vine. Rev. Elev. Méd. vét. Pays trop., 1964, 17 : 5-14.

11. PLOWRIGHT (W.) et FERRIS (R. D.). Studies with rinderpest in tissue culture. III. The stability of cultured virus and its use in virus neutralization tests. Arch. ges. Virusf., 1961, 11: 516-533.

12. PLOWRIGHT (W.) et FERRIS (R. D.). Studies with rinderpest virus in tissue culture. The use of attenuated culture virus as a vaccine for cattle. Res. vet. Sci., 1962, 3: 172-182.

13. PROVOST (A.). Rapport annuel de la Région de Recherches d'Afrique centrale, laboratorre de Farcha, pour 1968, pp. 116-117.

14. PROVOST (A.). Rapport annuel de la Région de Recherches d'Afrique centrale, laboratoire de Farcha, pour 1970, p. 124.

15. PROVOST (A.). Activité thermoprotectrice de la solution molaire de sulfate de magnésium sur l'inactivation thermique de Mycoplasma mycoides en phase liquide. C.R. Acad. Sci., Paris, 1970, 270 D : $3156-3157$

16. PROVOST (A.). Recherches immunologiques sur la péripneumonie. XIV. Description de deux techniques applicables sur le terrain pour le diagnostic de la maladie. Rev. Elev. Méd. vét. Pays trop., 1972, 25 : 475-496.

17. PROVOST (A.), BORREDON (C.) et BOCQUET (P.). Un vaccin mixte trivalent contre la peste bovine, la péripneumonie et le charbon bactéridien. Rev. Elev. Méd. vét. Pays trop. (à paraître).

18. PROVOST (A.), BORREDON (C.) et QUEVAL (R.). Recherches immunologiques sur la péripneumonie. XI. Un vaccin vivant mixte antibovipest1que-antipéripneumonique inoculé en un seul temps. Conception, production, contrôles. Bull. Off. int. Epiz., 1969, 72 a : 165-203.

19. PROVOST (A.) et QUEVAL (R.). Note sur l'hétéro-agglutinabilité des hématies de chèvres par les sérums de zébus. Ann. Inst. Pasteur, 1963, 105: $109-110$.

20. QUEVAL (R.), PROVOST (A.) et VILLEMOT (J. M.). Comparaison de méthodes de déviation 
du complément utilisées dans l'étude de la péripneumonie bovine. Bull. epiz. Dis. Afr., 1964, 12 : $159-170$.

21. RENOUX (G.) et RENOUX (M.). Effet immunostimulant d'un imidothiazole dans I'immunisation des souris contre l'infection par Brucella abortus. C.R. Acad. Sci., Paris, 1971, 272 D : 349-350.

22. RENOUX (G.) et RENOUX (M.). Action immunostimulante de dérivés du phénylimidothiazole sur les cellules spléniques formatrices d'anticorps. C.R. Acad. Sci. Paris, 1972, 274 D : 756-757.

23. SINGH (K, V.), OSMAN (O.A.), BAZ (T. I.) et
EL CICY (I. F.). The use of tissue culture rinderpest vaccine for egyptian cattle and water buffaloes. Cornell Vet., 1967, 57 : 465-479.

24. STAMATIN (N.). Recherches récentes et conceptions nouvelles sur la fièvre charbonneuse. II. La capacité immunisante de certaines substances sécrétées in vitro par B. anthracis. Rec. Méd. vét., 1964, 140 : 735-753.

25. THIENPONT (D.). Communication personnelle.

26. THIENPONT (D.) et collab. Tetramisole (R 8299) a new potent broad spectrum anthelmintic. Nature, 1966, 204 : 1084-1086. 